\title{
MÉMOIRES ORIGINAUX
}

\author{
MORPHOLOGIE ET COMPORTEMENT \\ DES FOURMIS LESTOBIOTIQUES \\ DU GENRE EPIXENUS EMERY \\ par \\ Francis BERNARD
}

En 1908, EMERy créait le genre Epixenus pour l'E. andrei Em., trouvé en Palestine dans un nid de Monomorium venustum Sm., et décrit sommairement par ANDRÉ (1881) comme une $q$ anormale de ce Monomorium. Epixenus est d'ailleurs très voisin des Monomorium, dont il diffère surtout par les pétioles des , très dilatés en écaille. En 1910, Forel décrivait $E$. biroi, sur des $q$ prises dans le nid de Monomorium creticum Em. (= Salomonis L.).

Cette particularité des pétioles caractérisant presque toujours des Myrmicinés parasites ou lestobiotiques, EMERY rangeait les Epixenus dans cette catégorie et, dans son Genera (1922) écrivait (p. 185) "Ouvrière inconnue; elle n'existe vraisemblablement pas. "

En 1942, ma première année en Algérie, je découvris assez communément, aux environs d'Alger, une forme nouvelle qui va être décrite ci-dessous et dont les ouvrières étaient abondantes. Des observations plus récentes (mai 1955) permettent d'affirmer que cette espèce n'est pas toujours lestobiotique et possède le plus souvent des nids indépendants bien constitués. En outre, un autre Epixenus inédit ( $E$. guineensis Bernard) avait été décrit en 1952 sur des ouvrières récoltées par M. LAмотTE aux monts Nimba (Guinée).

Donc, Epixenus possède les trois castes, au moins chez 2 des 5 espèces actuellement admises, et est aussi bien éthiopien que méditerranéen du Sud. Il m'a semblé utile, au stade actuel de nos connaissances, de publier une courte monographie de ce genre, très peu connu encore, avec le résumé des observations sur $E$. algiricus n. sp., seule forme suivie sur le terrain, la seule aussi dont les larves aient été capturées :

INSECTES SOCIAUX, TOME II, No 4, 1955. 
I' DIAGNOSE D'EPIXENUS ALGIRICUS N. SP. (fig. I)

\section{OUvrières :}

Types. - 12 ợ prises dans le même nid à Kaddous, à 7 kilomètres au sud d'Alger, sur une colline à Lentisques et argile en forte pente (altitude 150 mètres). Mars 1945.

Cotypes. - Plus de 200 ఛ prises, soit à Kaddous, soit dans la forêt de Baìnem, à 380 mètres d'altitude, à 6 kilomètres à l'ouest d'Alger, sol argileux horizontal. Les $\breve{+}$ sont très uniformes et diffèrent très peu d'une station à l'autre. 1947 à 1955.

Taille : $2^{\mathrm{mm}}, 2 \dot{\mathrm{a}} 2^{\mathrm{mm}}, 5$ (tandis que les races locales de Monomorium salomonis (L.) ont toutes de $2^{\mathrm{mm}}, 5$ à $2^{\mathrm{mm}}, 8$ ). L'allure et la démarche sont tout à fait celles de ce Monomorium.

Dessus du corps bien luisant, d'un brun foncé presque noir chez les individus âgés, d'un brun rougeâtre chez les immatures. Les fémurs et les scapes sont presque aussi foncés. De teinte plus claire sont les funicules, les tibias et les tarses, tous d'un jaune foncé ou roux clair. Les mandibules sont jaune clair, avec leurs dents et un fin liséré au bord externe d'un brun noir. Dessous du corps brun clair.

Côtés de la tête en entier, son tiers postérieur dorsal et tout le thorax finement et élégamment réticulés. Entre les mailles de cette réticulation, très égales entre elles, le tégument est lisse et luisant. Les deux tiers antérieurs de la tête ont de fines stries longitudinales qui divergent en arrière vers les yeux et passent insensiblement à la réticulation très peu après le niveau des yeux. Il n'y a pas d'aire lisse au milieu de la tète, mais de l'aire frontale (petite cordiforme, lisse ou faiblement ponctuée) part vers l'arrière un court sillon lisse assez enfoncé, qui se termine par un point ovale enfoncé entre les milieux des yeux. Pétioles faiblement ponctués, gastre lisse et brillant, sauf à son bord antérieur, qui est un peu réticulé.

Pilosité très faible sur le corps : quelques grands poils raides, blane jaunâtre, sur le clypéus, le pétiole et le gastre; pas de pubescence. Pattes et appendices à courte pilosité blanchâtre, éparse.

Tête très convexe, longue de $0 \mathrm{~mm}, 75$ (sans les mandibules) et large de $0 \mathrm{~mm}, 65$ (chez $M$. salomonis, elle a en moyenne: longueur 0,80 , largeur $0,75)$. Bords subparallèles, à peine élargis au niveau des yeux, qui sont un peu en avant du milieu de la tête. Vertex rectiligne, non rebordé. Yeux ovoïdes, très peu convexes, de 55 à 65 facettes. Scape court, dépassant à peine le bord postérieur de la tète.

Funicule moyen, son premier article égal aux trois suivants réunis. Les articles 2 à 7 aussi larges ou un peu plus larges que longs, l'article 8 et ceux de la massue nettement plus longs que larges.

Dessus du thorax peu convexe, assez rétréci au niveau du sillon méso- 
épinotal, qui est large, superficiel et cannelé en long. Epinotum deux fois plus long que large, son bord postérieur rectiligne, ses angles postérieurs émoussés, sa face déclive un tiers plus courte que sa face antérieure.

Monomorium salomonis est très analogue, mais chez lui tous les articles $\mathrm{du}$ funicule sont bien plus longs que larges, la tête n'a pas de sillon longitudinal médian après l'aire frontale, le corps est bien moins luisant et, surtout, les pétioles sont plus étroits (fig. 1,e).

Le pétiole possède un pédoncule aplati, roux, translucide, aussi long que la moitié postérieure élargie. Celle-ci, aussi large que longue, est très convexe en dessus, mais à angle supérieur émoussé.

Post-pétiole environ d'un cinquième plus large que le pétiole; son nœud, de même profil que le premier nœud, est également noir et faiblement ponctué.

Femelles (reines désailées prises dans les nids) :

Types. - 7 reines de Kaddous, d'une seule fourmilière très peuplée. Mars 1945.

Cotypes. - 4 reines de la forêt de Bainem, très semblables aux types. Une seule ㅇ dans chacun des nids fouillés, sauf un nid contenant 2 reines, mais il $\mathrm{y}$ en avait peut-être d'autres en profondeur.

Taille : $3^{\mathrm{mm}}, 8$ à $4^{\mathrm{mm}}, 1$ (les reines de $M$. salomonis ont $5^{\mathrm{mm}}, 2$ à $5^{\mathrm{mm}}, 8$, elles sont donc relativement plus grandes que les $\breve{q}$ par rapport à Epixenus). Le volume du gastre de la reine de Monomorium est plus que double de celui d'Epixenus.

Couleur très analogue à celles des $\overleftarrow{+}$, mais pilosité plus forte, épinotum plus anguleux, pétioles encore plus dilatés et funicule légèrement plus allongé. La teinte générale est d'un noir plus foncé que celui des $\overline{+}$ àgées, et la cuticule est plus lisse.

Tête subcarrée, bien moins sculptée que chez l'市: quelques stries longitudinales effacées vers le milieu du tiers antérieur, une vague réticulation aux angles postérieurs, le reste presque lisse.

Yeux très peu convexes, d'environ 110 facettes, placés très peu en avant du milieu de la tête. Ocelles arrondis, une légère dépression triangulaire entoure l'ocelle antérieur.

Comme chez l'⿳亠口, un court et large sillon médian part de l'aire frontale et sa longueur est sensiblement égale à celle d'un œeil. Ce sillon a un peu la forme d'un point d'exclamation renversé. Vertex faiblement concave.

Le scape n'attẹint pas, en arrière, le bord postérieur de la tête. Au funicule, les articles 2 à 7 sont un peu plus longs; aucun n'est plus large que long, mais presque tous sont subcarrés. Thorax plus luisant, plus convexe et bien plus élargi vers l'avant que celui de Monomorium. Surface ornée d'une réticulation à peine visible, effacée. Pronotum très convexe, arrondi, presque deux fois plus large que l'épinotum. Mésonotum ovale, plan en dessus, 
2,5 fois plus long que large. Métanotum rougeâtre, aussi large que long, avec une forte gibbosité longitudinale tout le long de son tiers médian.

Segment médiaire aussi nettement réticulé que chez l'ŏ. L'épinotum, subtriangulaire en dessus, a sa moitié antérieure convexe et sa zone postérieure un peu concave. Angles postérieurs très marqués, arrondis, mais relevés et légèremenl dentiformes. Pétioles proportionnellement un tiers plus larges que ceux de l'ఢ̆, leurs nœuds dilatés en écailles, mais le sommet de ces écailles est tronqué, émoussé, finement et peu densément ponctué. Elles sont loin d'être aussi tranchantes que les écailles des Formicinx.

a.

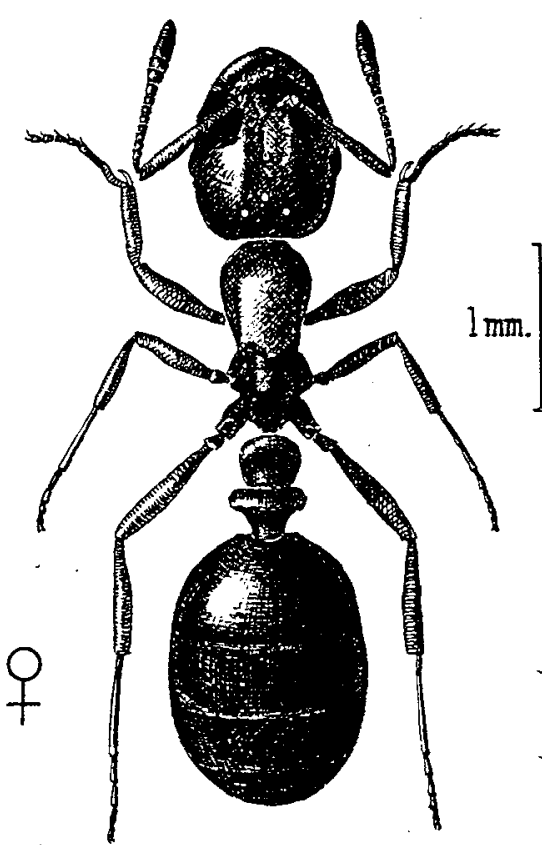

b.

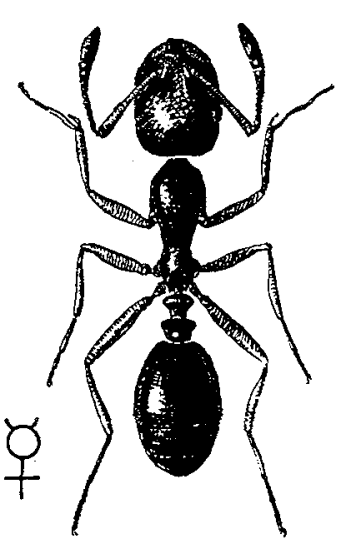

d.

e.<smiles></smiles>

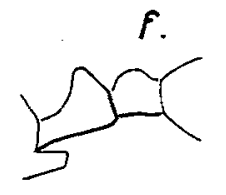

c.
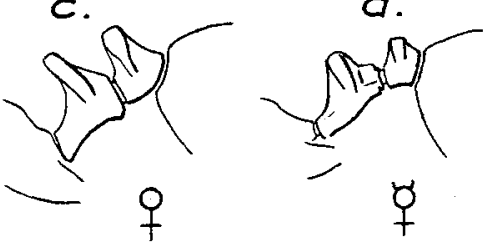

CA

Fig. 1. - Epixenus algiricus n. sp., des environs d'Alger. - a, reine, de la forêt de Baïnem, longueur 4 millimètres; $b$, ouvrière, longueur $2^{\mathrm{mm}}, 3 ; c$, pétioles de la reine, vus de profil ; $d$, pétioles de l'ouvrière; $e$, pétioles de face et, $f$, de profil, chez une ouvrière de Monomorium salomonis (L.) [voisine d'Epixenus et partois pillée par lui].

Pilosité bien plus développée que chez l'ọ. Vers chaque angle postérieur de la tête, un grand poil tactile blanc, dressé, avec fossette piligère. Sur le métanotum et sur chacun des pétioles, 4 à 12 poils analogues, accompagnés de plus petits. Il y en a 16 à 20 en arrière de chaque tergite abdominal. Sur la tête, une pilosité banale courte, très espacée, blanchâtre. Poils couchés des appendices un peu plus serrés que ceux des ouvrières.

En résumé, les reines sont d'un tiers plus courtes que celles de Monomorium et d'aspect bien différent. Elles sont agiles, peu craintives, et il doit y en avoir plusieurs dans les fourmilières âgées, comme celle de Kaddous 
où j'avais pris huit femelles fécondes. Cette polygynie est également le cas chez la plupart des Monomorium nord-africains.

\section{Males :}

Aucun mâle d'Epixenus n'est décrit avec certitude. E. creticus (EMERY, 1908) est basé sur un ${ }^{t}$ de Crète, et son appartenance à ce genre est douteuse. De mon côté, j'ai pris au fort des Arcades, au-dessus d'Alger, un ô pouvant se rattacher à Epixenus, mais il est très fortement ponctué et plus

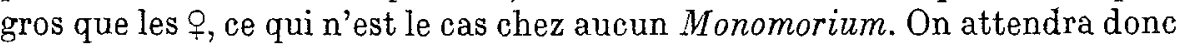
des captures authentiques dans le nid pour identifier ce sexe.

\section{$2^{\circ}$ MORPHOLOGIE LARVAIRE D'EPIXENUS ALGIRICUS (fig. 2)}

Les larves de Fourmis sont encore mal connues, si on les compare aux larves de Sphégides, d'Abeilles, de Vespides, qui ont fait l'objet de nombreuses monographies. Bien des espèces et même des genres classiques de la région méditerranéenne n'ont pas encore leurs larves décrites.

Pourtant, malgré l'uniformité apparente de ces stades apodes, W. M. WhEELER (1918) avait raison d'insister sur leur intérêt biologique. Il a été le premier à montrer que la structure larvaire varie beaucoup avec les tribus et que des sous-familles évoluées comme les Formicinæ ont encore des larves primitives.

De 1923 à aujourd'hui paraissent les publications très précises de G. C. Wheeler, apportant d'excellentes figures sur de nombreux genres américains. Dans mon propre laboratoire, H. Gantés (1949) a fait une bonne étude sur les larves de seize genres d'Algérie et leur croissance. C. Athias-Henriot (1948) et S. Valentini (1951) ont contribué fructueusement à l'anatomie des larves.

De tous ces travaux (environ 14 références de valeur sur ce sujet), on peut déduire que l'étude des larves aidera beaucoup à comprendre l'évolution et l'écologie des Fourmis. Généralement, leur morphologie est assez différente d'une espèce à l'autre pour que l'on puisse établir (plus tard) des tableaux de détermination des larves, basés surtout, dans un même genre, sur la pilosité du corps et la structure des pièces buccales. L'anatomie et la croissance sont assez variables selon les espèces pour qu'elles jouent un rôle incontestable dans l'adaptation des Fourmis au milieu. C. Athias-HenRiот a prouvé que les trois Messor examinés par elle ont des conformations très diverses du tube digestif larvaire. $H$. GanTÉs a montré que certaines formes du désert (Monomorium gracillimum, Cataglyphis albicans) ont des larves néonates d'une grosseur exceptionnelle, adaptation probable à l'aridité locale par une moindre surface relative de leur corps.

Je dispose jusqu'à présent de deux séries de larves d'Epixenus, prises en mai 1955 dans les nids de la forêt de Baïnem :

3 grosses larves de $1 \mathrm{~mm}, 7$ à $1 \mathrm{~mm}, 9$; 12 larves de $1 \mathrm{~mm}, 15$ à $1 \mathrm{~mm}, 30$. 
Par comparaison avec Monomorium salomonis, qui a cinq stades larvaires comme la plupart des Fourmis, il est probable que les petites larves représentent le stade 2 et les grosses le stade 3. A part la taille, leurs morphologies sont très semblables (fig. 2) :

Tête bien différenciée, au moins autant que chez une larve de Monomorium, avec mandibules jaunes très visibles. Corps blanc au stade 2, jaunâtre ou brûnatre au stade 3 . Les 3 segments thoraciques ont leurs limites

a.

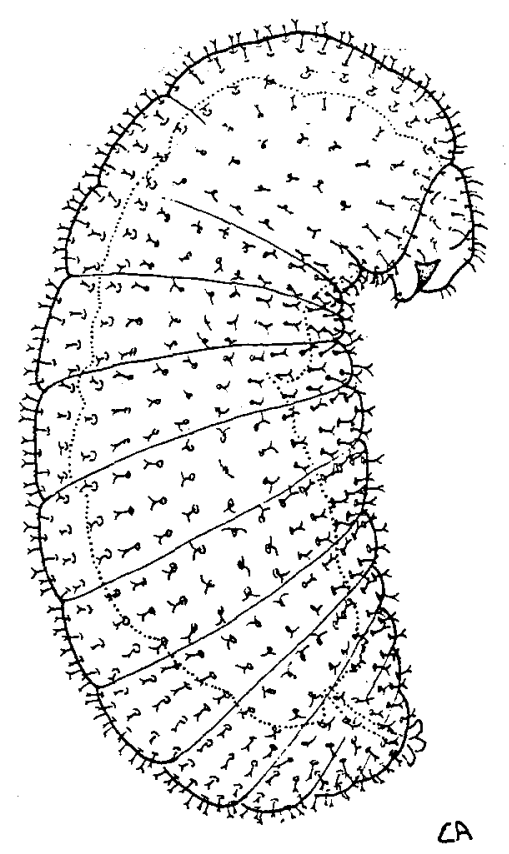

b.
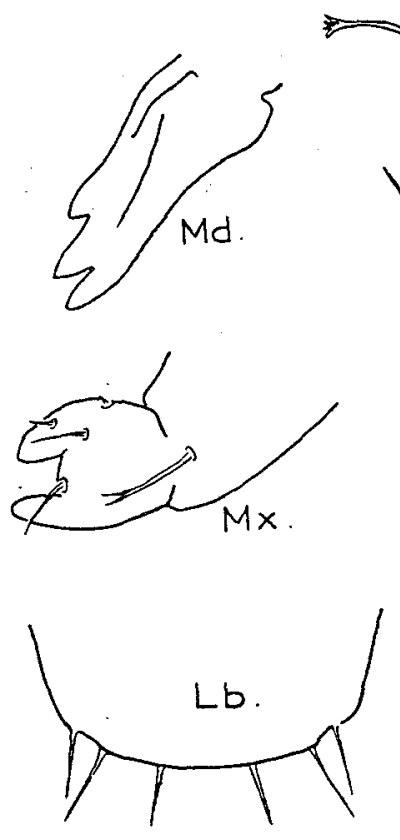

c.<smiles>CC(C=[Co])=C[Te]</smiles><smiles>CC(C)=O</smiles>

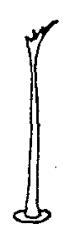

Fig. 2. - Epixenus algiricus $\mathrm{n}$. $\mathrm{sp}$. : larve de $1^{\mathrm{mm}}, 2$ probablèment au deuxième stade. - $a$, larve de profil ; on remarquera l'uniformité des poils et la séparation des segments, bien plus nette que chez Monomorium; $b$, pièces buccales, $\times 200$. Les mandibules sont jaune foncé, les autres pièces incolores et munies de poils simples; $c$, les quatre principales formes de poils du corps, $\times 250$. Ils sont presque tous bifurqués. Le deuxième type, à partir du haut, à pointes non ramiflées ni dentées, est de loin le plus fréquent.

peu distinctes sur la face dorsale et très difficiles à voir ventralement. Les dix segments abdominaux ont leurs séparations bien nettes dorsalement et ventralement, mais non prolongées sur les côtés, sauf à la bordure du pygidium.

Tous les segments et la tête portent de nombreux poils courts, dressés et plus ou moins incurvés. Seule la région des pièces buccales est peu poilue, avec quelques poils isolés rectilignes trois fois plus courts que les autres. Presque tous les poils du corps et du dessus de la tête sont courts, bifurqués, 
à branches de la fourche incurvées. Cela rappelle beaucoup $M$. salomonis, mais, chez ce dernier, il y a en plus un cercle de grands poils, rectilignes, en avant du prothorax.

Ainsi faites, ces larves diffèrent notablement de celles des Monomorium déjà connus (M. salomonis (L.) et gracillimum Sm.) par leur corps moins cylindrique, bien plus poilu, les limites des segments beaucoup mieux indiquées, surtout pour les segments abdominaux 4 à 10. Le bord antérieur du prothorax ne porte pas de couronne de grands poils comme chez Monomorium.

Les types larvaires les plus voisins seraient ceux de Leptothorax acervorum Nyl., décrit par H. Gantés (1949, fig. 6, pl. V), et de Pheidole dentata Mayr, figuré par G. C. Wheeler (1953, fig. 11, pl. III). Encore ce Pheidole et ce Leptothorax ont-ils des poils larvaires plus longs et plus variés que notre Epixenus.

En somme, la larve d'E. algiricus est moins évoluée, moins simplifiée extérieurement que celles des Monomorium, ce qui correspond aux caractères des adultes, où la reine surtout est moins comprimée et moins différente des ouvrières que chez Monomorium. Le genre Epixenus est donc, à divers égards, plus primitif que son proche parent Monomorium. Si les ouvrières amènent à le rapprocher étroitement de Monomorium, les larves s'éloignent de la tribu des Solenopsidini et peuvent être comparées à celles des Leptothoracini, sauf pour la pilosité.

\section{$3^{\circ}$ NOTES SUR LE COMPORTEMENT DES EPIXENUS}

La première forme décrite, $E$. andrei Em., est basée sur des ㅇ mêlées à un nid complet de $M$. venustum Sm., en Palestine. C'est tout ce qu'on en sait, et ce détail, joint aux pétioles dilatés, faisait considérer par EMERY les Epixenus comme des Fourmis parasites, très probablement dépourvues d'ouvrières.

Cette question est entièrement à reconsidérer depuis la découverte à Alger de nombreux nids pleins d'ouvrières normales, non mélangés à des fourmilières d'autres espèces. Nos observations, faites surtout en avril 1945 et en mai 1955, sont résumées ici et devront être complétées plus tard :

\section{ÉCOLOGIE :}

Aux environs d'Alger, Epixenus algiricus habite surtout l'argile pure, où il creuse des nids étroits, simples, peu profonds. Ces nids sont assez rares sur les collines à Lentisques (Kaddous, Ben Aknoun), relativement communs en forêt de Baïnem, boisée de Pins d'Alep et située de 300 à 400 mètres d'altitude, près de la Méditerranée, à 6 kilomètres d'Alger. A Baïnem, les terrains étudiés sont horizontaux ou presque. L'argile, jaunâtre, est très mêlée de gravier (quartz et gneiss) et se trouvait presque sèche lors du relevé 
suivant, qui donnera une notion moyenne de l'habitat et des principales Fourmis concurrentes :

Relevé $n^{0}$ 147. - Route forestière allant de la forêt de Baïnem à Guyotville. 12 mai 1955. Les fourmilières logent surtout dans l'argile dénudée des bords de la route, larges de 1 à 2 mètres. Plantes dominantes sur ces bordures : Trifolium et Asphodèles ; dans la forêt : Cistes et Quercus coccifera. La rareté des Mollusques, des Lampyris et des Cloportes montre que cette station est relativement sèche, d'ailleurs loin de toute mare.

Voici, par ordre d'abondance décroissante, les nombres de nids des principales Fourmis, pour un total de 100 fourmilières repérées :

Tetramorium punicum (Sm.): 18. - Tapinoma simrothi Krausse : 14. - Epixenus algiricus Bernard : 12. - Cataglyphis viatica Först. : 12. - Messor barbara (L.) : 10. - Aphænogaster gemella (Rog.): 8. Camponotus barbaricus (Em.) : 8 .

Donc, ici, Epixenus vient au troisième rang par ordre d'abondance, et ses nids sont aussi fréquents que ceux de Cataglyphis viatica, espèce très banale sur ces sols argileux à Bainem, à Tunis et au Maroc. Ces Fourmis seraient sans doute encore plus abondantes si elles n'étaient concurrencées sur place par Tapinoma simrothi, importé d'Orient et très nuisible aux jardins d'Algérie.

Epixenus semble surtout insectivore, comme Monomorium salomonis, mais certains nids contenaient des débris de feuilles et de pétales de Ciste. Une ouvrière transportait un Hémiptère Capside tué.

\section{STRUCTURE DES NIDS :}

Les 24 fourmilières d'E. algiricus déjà explorées sont d'un type assez uniforme. Chacune s'ouvre à la surface de l'argile par un petit trou de 1 à 3 millimètres, peu visible. 1 à 2 centimètres après ce trou, on trouve déjà des larves, assez dispersées et non classées par rang de taille. Au plus 2 ou 3 courtes galeries divergentes, ne descendant guère qu'à 5 centimètres sous l'ouverture, contiennent reines, ouvrières et d'autres larves. Un nid à Kaddous contenait au moins 8 reines; les autres, probablement une reine ou 2 au maximum. Mais les ouvrières étaient toujours nombreuses, souvent plus de 500 , et une fois plusieurs milliers.

L'allure et la vitesse des ouvrières sont très analogues à celles de $M$. salomonis. Les reines sont moins craintives et beaucoup moins cachées que chez la majorité des Fourmis locales. Malgré l'éventration du nid, plusieurs reines se promenaient au soleil dans le trou pratiqué et ne descendaient pas dans les galeries. Ce comportement indifférent caractérise aussi d'autres reines d'espèces polygynes, comme les Monomorium et les Tapinoma.

\section{LESTOBIOSE ÉVENTUELLE :}

Sur 24 nids d'Epixenus bien fouillés, il y en avait au moins 18 (75\%) entièrement indépendants, isolés à plus de 2 mètres de toute autre four- 
milière et ne communiquant pas avec elle. 6 autres nids (25\%) étaient exactement au-dessus d'une fourmilière plus grande, avec mélange partiel des $\breve{+}$ des 2 espèces :

- 2 avec Messor barbara (L.);

- 1 avec Messor sancta (Forel);

- 2 avec Camponotus sylvaticus barbaricus $\mathrm{Em}$;

- 1 avec Monomorium salomonis (L.), à Kaddous.

Dans ces cas de mélange, le nid superficiel d'Epixenus ne contenait aucune larve et le couvain était peut-être mêlé à celui de l'hôte ? Je n'ai pourtant pas réussi à distinguer de petites larves d'Epixenus parmi celles, 2 à 3 fois plus grosses, des Messor et Camponotus.

De tels nids directement superposés, avec mélange plus ou moins net des populations, rappellent d'autres espèces considérées comme "lestobiotiques " ou voleuses. Telles sont, en Europe, la plupart des Solenopsis. Il ne faut toutefois pas exagérer les habitudes pilleuses ou inquilines de ces minuscules Fourmis. Forel les considérait comme toujours mêlées à d'autres genres, mais aussi bien à Banyuls (pour Solenopsis Emeryi banyulensis) que dans les Alpes (pour $S$. monticola Bernard), j'ai noté de nombreux nids homogènes, richement peuplés et fort éloignés de toute autre fourmilière. Dans les Alpes-Maritimes, $S$. nicæensis Bernard est tantôt associé à Camponotus æthiops Latr., tantôt manifestement indépendant. De même, les petits Plagiolepis ( 3 espèces en France et 5 en Afrique du Nord) sont souvent indépendants, parfois mêlés à de gros nids de Camponotus dont les habitants semblent indifférents à leur hôte de faible taille. Par contre, les Fourmis de dimensions plus réduites (Pheidole, Monomorium, Tetramorium...) perçoivent fort bien les Plagiolepis et Solenopsis et cherchent généralement à s'en débarrasser.

En résumé, Epixenus algiricus, comme bien des Solenopsis, forme très souvent des nids indépendants. Quand il est mélangé avec d'autres Fourmis, sans doute pille-t-il plus ou moins leur société, mais il ne semble pas très nuisible et la reine comme les larves de l'hôte ont alors une allure normale.

\section{TABLEAU DES Epixenus DÉJA DÉCRITS :}

Les 2 premières espèces : $E$. andrei Em. et $E$. biroi Forel ne sont établies que sur des + . J'y ajouterai la reine d'E. algiricus et donnerai un autre tableau pour les 2 ouvrières connues, d'ailleurs bien différentes l'une de l'autre. Le $\widetilde{\sigma}$ (E. creticus Em.) est encore trop douteux pour en tenir compte ici.

\section{TABLEAU Des Femelles :}

(Les 3 formes signalées se ressemblent beaucoup quant à la structure des antennes et des pétioles, qui, par conséquent, ne seront pas utilisés.) 
1. Reine très lisse et très luisante, à pilosité jaune roussâtre. Dents postérieures de l'épinotum très larges, saillantes. Le mésonotum, étroit, rappelle celui de femelles ergatomorphes. Roux brun, $3^{\mathrm{mm}}, 5 \mathrm{à} 4 \mathrm{~mm}, 2$. Crète, avec Monomorium ......

- Reines en partie ponctuées ou réticulées, à pilosité blanchâtre. Dents de l'épinotum nettes, mais arrondies et peu saillantes. Mésonotum normal ............................

E. biroi Forel.

2. Tête plus large que longue. Épinotum mat, très ponctué. $4^{\mathrm{mm}}, 3$, rousse. Palestine, avec Monomorium venustum $\ldots \ldots \ldots \ldots \ldots \ldots \ldots \ldots$

- Tête subcarrée. Épinotum assez luisant, réticulé. $3 \mathrm{~mm}, 8$ à $4 \mathrm{~mm}, 1$, brun foncé ou noire. Environs d'Alger, libre ou voisin d'autres Fourmis. . .

E. andrei Em.

E. algiricus Bernard.

TABLEAU DES OUVRIÉRES :

- Taille $2^{\mathrm{mm}}, 2$ à $2^{\mathrm{mm}}, 5$. Brun noir, appendices roux. Deuxième article du funicule égal à 3 et 4 , tous à peine plus larges que longs. Mésonotum peu convexe. Épinotum sans dents nettes .....

- Taille $1 \mathrm{~mm}, 8$ à $1 \mathrm{~mm}, 9$. Noires à appendices jaunes. Deuxième article du funicule très court, plus que 3 et 4 , qui sont bien plus larges que longs. Mésonotum très convexe. Dents postérieures de l'épinotum très visibles............

E. algiricus Bernard.

E. guineensis Bernard, monts Nimba (Guinée).

\section{Résumé.}

Les petites Fourmis du genre Epixenus, considérées comme parasites et

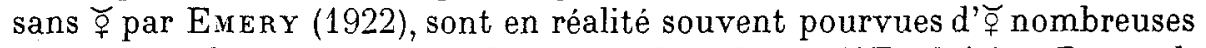
et capables de nicher indépendamment. C'est le cas d'E. algiricus Bernard, décrit ci-dessus, qui peut avoir plus de 1000 ouvrières et 8 reines. Le quart seulement des nids observés aux environs d'Alger semblaient associés à d'autres genres, surtout Messor et Camponotus. Les Epixenus (4 espèces certaines) sont surtout insectivores et assez proches des Monomorium, avec toutefois des larves et des reines plus primitives.

\section{Summary.}

The small Ants of the genus Epixenus, described by Emery (1908) as probably parasitic and without workers, are in fact often with numerous workers and have independent nests. The best known is $E$. algiricus Ber- 
MORPHOLOGIE ET COMPORTEMENT DES FOURMIS LESTOBIOTIQUES 283

nard, described here, having sometimes more than 1000 ○ and 8 queens. Only one fourth of the nests observed in the neighbourhood of Algiers seems to be associated with other genera, chiefly Messor and Camponotus. Epixenus (4 sure species) are mainly insectivorous and near Monomorium, with, however, queens and larvae more primitives.

\section{BIBLIOGRAPHIE.}

1891. ANDrÉ (E.). - Species des Hyménoptères d'Europe et d'Afrique du Nord, p. 335, pl. XXII. Beaune.

1949. Bernard (F.). - Notes sur les Fourmis de France. II : Peuplement des montagnes méridionales (Ann. Soc. Ent. Fr., vol. CXV, 1949, p. 1-36). - 1952. La réserve naturelle intégrale du mont Nimba, XI. Hyménoptères Formicidæe (Mémoires de l'I. F. A. N., no 19, p. 238).

1908. EM ERy (C.). - Beiträge zur Monographie der Formicidae des Palaearktischen Faunengebietes (Deutsche Entom. Zeitschrift, 1908, p. 558). - 1922. Genera Insectorum, vol. 174 B, p. 185, Bruxelles.

1910. Forel (A.). - Ann. Soc. Entom. Belgique, vol. 54, p. 21.

(Laboratoire de Zoologie. Faculté des Sciences d'Alger.) 